\title{
Unifying Brillouin scattering and cavity optomechanics in silicon photonic wires
}

\author{
Raphaël Van Laer, Bart Kuyken, Roel Baets and Dries Van Thourhout \\ Photonics Research Group, Department of Information Technology, Ghent University - imec \\ $\mathcal{E}$ Center for Nano- and Biophotonics, Ghent University \\ raphael.vanlaer@intec.ugent.be
}

\begin{abstract}
We prove a connection between the Brillouin gain and the zeropoint optomechanical coupling rate. Moreover, we report on observations of Brillouin scattering in silicon photonic nanowires - showing efficient coupling between near-infrared light and gigahertz sound.
\end{abstract}

(C) 2014 Optical Society of America

OCIS codes: $130.4310,190.4390,290.5900$.

Stimulated Brillouin scattering (SBS) has seen rising interest with the promise of chip-scale alloptical functionalities and potential applications in microwave signal processing. ${ }^{1}$ The prediction of strong Brillouin gain coefficients (up to $10^{5} \mathrm{~W}^{-1} \mathrm{~m}^{-1}$ ) in fully suspended silicon waveguides ${ }^{2,3}$ was central to this evolution. We recently made the first observation of SBS in silicon photonic nanowires, ${ }^{4}$ showing on/off gain up to $175 \%$ (fig.1). These wires thus emerge as a promising SBS platform. We report theoretical and experimental progress towards actual Brillouin devices, including lasers and comb generators, based on these nanowires.

For most of their history, Brillouin scattering ${ }^{5}$ and cavity optomechanics ${ }^{6}$ have been disconnected branches of research. Although both study the interaction between light and sound, a number of differences impeded their unification. For instance, SBS typically deals with counterpropagating and cavity optomechanics with co-propagating pump and Stokes waves. In addition, electrostrictive bulk forces usually dominate sound generation in SBS - while radiation pressure at the boundaries takes this role in cavity optomechanics. Further, cavity optomechanics typically studies resonators with much larger optical than mechanical loss rates $\left(\kappa \gg \Gamma_{\mathrm{m}}\right)-$ whereas Brillouin lasers ${ }^{7}$ operate in the reversed regime $\left(\Gamma_{\mathrm{m}} \gg \kappa\right)$.

In the last years, the idea that these are mostly superficial differences has been gaining traction. Indeed, in both cases light generates motion and the motion phase-modulates light. Next, this spatiotemporal phase-modulation creates motional sidebands. Brillouin scattering and cavity optomechanics share this essential feedback nature. Some progress towards fusion of SBS and cavity optomechancs has already been made. For instance, electrostrictive forces were exploited for sideband cooling ${ }^{8}$ and radiation pressure contributed to SBS in nanoscale silicon waveguides. ${ }^{4,9}$ Here we take another step in the Brillouin-optomechanics fusion.

Theoretically, we derive the cavity dynamics from the waveguide equations of motion in the high-finesse limit. The detailed transition will be published fully elsewhere. It was inspired by the derivation of the mean-field Lugiato-Lefever equation from the nonlinear Schrödinger equation in the context of Kerr microcombs. ${ }^{10}$ This explicit transition shows the unity of optomechanical phenomena, regardless of whether they occur in waveguides or cavities. Further, it automatically links two parameters of central importance in SBS and cavity optomechanics: respectively the SBS gain coefficient $G_{\mathrm{SBS}}\left[\mathrm{W}^{-1} \mathrm{~m}^{-1}\right]$ and the zero-point coupling rate $g_{0}[\mathrm{~Hz}]$. The former $\left(G_{\mathrm{SBS}}\right)$ quantifies the pump power and waveguide length required to significantly amplify a Stokes seed. The latter $\left(g_{0}\right)$ quantifies the cavity frequency shift induced by the mechanical zero-point motion. We prove that these parameters are inextricably linked by the identity

$$
g_{0}^{2}=v_{\mathrm{g}}^{2} \frac{\left(\hbar \omega_{0}\right) \Omega_{\mathrm{m}}}{4 L_{\mathrm{m}}}\left(\frac{G_{\mathrm{SBS}}}{Q_{\mathrm{m}}}\right)
$$

with $v_{\mathrm{g}}$ the optical group velocity $[\mathrm{m} / \mathrm{s}], \hbar \omega_{0}$ the photon energy $[\mathrm{J}], \frac{\Omega_{\mathrm{m}}}{2 \pi}$ the mechanical resonance frequency $[\mathrm{Hz}], L_{\mathrm{m}}$ the mechanical oscillator length $[\mathrm{m}]$ and $Q_{\mathrm{m}}$ the mechanical quality factor [-]. The SBS-optomechanics connection is strongest for intra-modal forward SBS, but a completely 

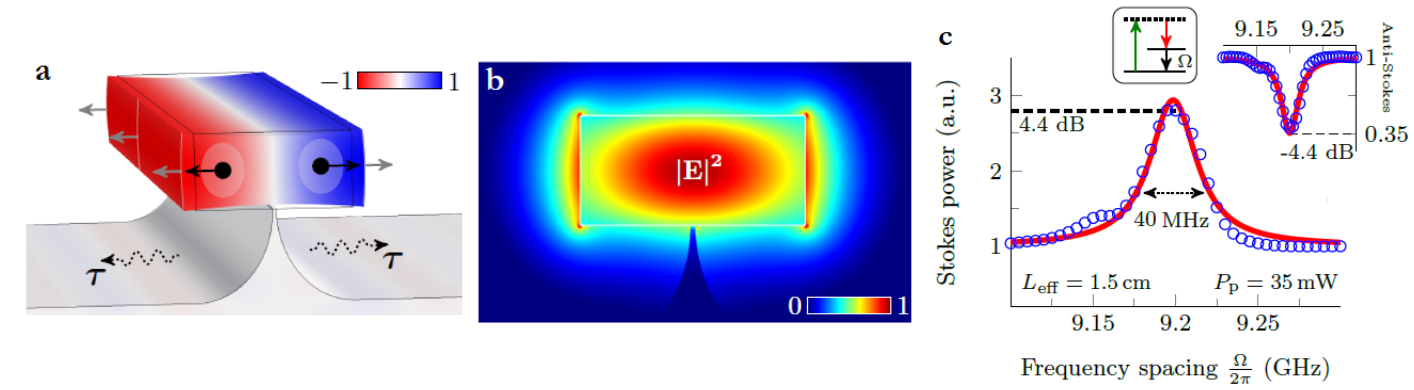

Fig. 1: a, The observed 9.2GHz acoustic mode aligns with the bulk electrostrictive forces (black arrows) and the boundary radiation pressure (grey arrows). The leakage of phonons through the pillar determines their lifetime $\tau \approx 5 \mathrm{~ns}$. $\mathbf{b}$, Electric field norm of the quasi-TE optical mode. $\mathbf{c}$, A Lorentzian gain resonance, obtained by scanning the frequency spacing $\frac{\Omega}{2 \pi}$ between a weak Stokes seed and a strong pump. The inset shows an identical depletion profile on an anti-Stokes seed.

analogous transition can be made for inter-modal forward and backward SBS. Equation (1) takes into account both electrostriction and radiation pressure.

We recently observed $2.3 \mathrm{~dB} / \mathrm{cm}$ on/off intra-modal forward SBS gain in silicon nanowires (fig.1) with $2.6 \mathrm{~dB} / \mathrm{cm}$ propagation loss. ${ }^{4}$ These partially suspended wires have a gain coefficient $\mathrm{G}_{\mathrm{SBS}}$ of $3100 \mathrm{~W}^{-1} \mathrm{~m}^{-1}$ at $\frac{\Omega_{\mathrm{m}}}{2 \pi}=9.2 \mathrm{GHz}$ with a linewidth of $\frac{\Gamma_{\mathrm{m}}}{2 \pi} \approx 35 \mathrm{MHz}$. They combine good phonon confinement with a centimeter-scale interaction length and a large photon-phonon overlap. Applied to these silicon nanowires, connection (1) shows that values of $\frac{g_{0}}{2 \pi} \approx 500 \mathrm{kHz}$ are in reach in $20 \mu \mathrm{m}$-roundtrip silicon microrings - comparable to the best coupling rates so far. ${ }^{6}$ Thus, relation (1) will be susceptible to experimental tests in the near future.

In conclusion, we prove a connection between the SBS gain coefficient and the zero-point optomechanical coupling rate. This link shows the unity of waveguide- and cavity-based optomechanical effects. In addition, we made the first observation of SBS gain in silicon photonic nanowires. This is a significant step towards optically-driven lasers/sasers, microwave filters and comb generators on a densely integrated silicon chip.

Acknowledgement. R.V.L. acknowledges the Agency for Innovation by Science and Technology in Flanders (IWT) for a PhD grant. This work was partially funded under the FP7-ERC-InSpectra programme and the ITN-network cQOM.

1. R. Pant et al., "On-chip stimulated Brillouin scattering for microwave signal processing and generation," Laser E Photonics Reviews, 2014.

2. P. Rakich et al., "Giant enhancement of stimulated Brillouin scattering in the subwavelength limit," Physical Review X, vol. 2, pp. 1-15, Jan. 2012.

3. R. Van Laer et al., "Analysis of enhanced stimulated Brillouin scattering in silicon slot waveguides," Optics Letters, vol. 39, pp. 1242-5, Mar. 2014.

4. R. Van Laer et al., "Interaction between light and highly confined hypersound in a silicon photonic nanowire," arXiv preprint 1407.4977 (under review), 2014.

5. B. Eggleton, C. Poulton, and R. Pant, "Inducing and harnessing stimulated Brillouin scattering in photonic integrated circuits," Advances in Optics and Photonics, pp. 536-587, 2013.

6. M. Aspelmeyer, T. J. Kippenberg, and F. Marquardt, "Cavity Optomechanics," Reviews of Modern Physics, June 2014.

7. A. Nunnenkamp et al., "Quantum-Limited Amplification and Parametric Instability in the Reversed Dissipation Regime of Cavity Optomechanics," Physical Review Letters, vol. 113, p. 023604, July 2014.

8. G. Bahl, M. Tomes, F. Marquardt, and T. Carmon, "Observation of spontaneous Brillouin cooling," Nature Physics, vol. 8, pp. 203-207, Jan. 2012.

9. H. Shin et al., "Tailorable stimulated Brillouin scattering in nanoscale silicon waveguides," Nature Communications, vol. 4, p. 1944, Jan. 2013.

10. S. Coen et al., "Modeling of octave-spanning Kerr frequency combs using a generalized mean-field Lugiato-Lefever model," Optics Letters, pp. 1-4, 2013. 